FACTA UNIVERSITATIS

Series: Mechanical Engineering Vol. 15, N ${ }^{0} 3,2017$, pp. 517 - 533

https://doi.org/10.22190/FUME161210012B

Original scientific paper

\title{
CRANIOFACIAL STRESS PATTERNS AND DISPLACEMENTS AFTER ACTIVATION OF HYRAX DEVICE: FINITE ELEMENT MODELLING
}

\author{
UDC 531/534:[57+61]
}

\author{
Sergei Bosiakov ${ }^{1}$, Anastasia Vinokurova ${ }^{2}$, Andrei Dosta ${ }^{3}$ \\ ${ }^{1}$ Belarusian State University, Minsk, Belarus \\ ${ }^{2}$ Rzeszow Technology University, Poland \\ ${ }^{3}$ Belarusian Medical State University, Minsk, Belarus
}

\begin{abstract}
Rapid maxillary expansion is employed for the treatment of cross-bite and deficiency of transversal dimension of the maxilla in patients with and without cleft of palate and lip. For this procedure, generally, different orthodontic appliances and devices generating significant transversal forces are used. The aim of this study is the finite-element analysis of stresses and displacements of the skull without palate cleft and the skull with unilateral and bilateral cleft after activation of the Hyrax orthodontic device. Two different constructions of the orthodontic device Hyrax with different positions of the screw relative palate are considered. In the first case, the screw is in the occlusal horizontal plane, and in the other, the screw is located near the palate. Activation of the orthodontic device corresponds to the rotation of the screw on one-quarter turn. It is established that the screw position significantly affects the distributions of stresses in skull and displacements of the cranium without palate cleft and with unilateral or bilateral palate cleft. Stresses in the bone structures of the craniums without cleft and with cleft are transferred from the maxilla to the pterygoid plate and pharyngeal tubercle if the screw displaces from the occlusal plane to the palate. Depending on the construction of the orthodontic appliance, the maxilla halves in the transversal plane are unfolded or the whole skull is entirely rotated in the sagittal plane. The stresses patterns and displacements of the skull with bilateral palate cleft are almost unchanged after activation of the orthodontic devices with different positions of the screw, only magnitudes of stresses and displacements are changed. The obtained results can be used for design of orthodontic appliances with the Hyrax screw, as well as for planning of osteotomies during the surgical assistance of the rapid maxillary expansion.
\end{abstract}

Key Words: Unilateral Palate Cleft, Bilateral Palate Cleft, Hyrax Screw, Stresses Pattern, Displacements

Received December 10, 2016 / Accepted May 03, 2017

Corresponding author: Sergei Bosiakov

Belarusian State University, Department of Theoretical and Applied Mechanics, Nezavisimosti avenue 4, 220030 Minsk, 4, Belarus

E-mail: bosiakovsm@gmail.com 


\section{INTRODUCTION}

Cross bite is the most common transversal anomaly of the dentition interposition requiring a prolonged active treatment. Its frequent cause is violation of maxilla growth, reduced chewing function or chewing on one side, as well as congenital cleft palate. Among the defects of the craniofacial complex and maxilla, according to the World Health Organization, the congenital cleft of the lip and the palate are dominant. Rapid maxillary expansion (RME) is one of the treatment stages of transversal discrepancy of maxilla. Fixed orthodontic devices (appliances), such as devices with the Hyrax screw or palatal distractors are recommended for the RME $[2,18]$. Among various types of orthodontic appliances the most hygienic ones are Hyrax devices. Moreover, devices of this type are less traumatic and more comfortable for the patients besides having a low incidence of complications after application. At the same time, the design features of the orthodontic appliances for RME, including Hyrax devices, significantly affect the intensity and nature of motions of the skull bone structures and teeth [8, 9, 19, 23].

The finite-element $(\mathrm{FE})$ modeling is the main approach to understanding the influence of RME on the loads distribution in the cranium. The FE analysis of the orthodontic loads distributions during RME of the craniofacial complex without palate cleft was performed in $[3,6,7,10-13,17]$. An extensive review of the FE calculations of stresses and displacements of the maxillary complex under the action of different types of the orthodontic appliances was carried out in the recent study [13]. It should be noted that the common simplifying assumption adopted in the above-mentioned studies and other similar research projects is a simulation of the orthodontic appliance impact on the skull bone structures and teeth by means of the application of transversal displacements or forces to the anchor teeth. The FE analysis of RME effect on skull with the unilateral cleft palate was carried out in [16]. In this study the distributions of transversal forces in the craniofacial complex with the unilateral palate cleft were evaluated as well as their influence on the displacements of the naso-maxillary bones. To simulate the clinical situation the displacements of $5 \mathrm{~mm}$ in transversal plane to the maxilla premolars and first molars were applied. Assessment of the RME impact for patients with unilateral cleft palate using the FE method was performed in [4]. This study was a preliminary step in the development of surgical techniques for RME assistance. In accordance with [4], for effective RME of the skull with unilateral cleft the osteotomy of the median palatal suture and of the lateral buttress is usually required for an effective maxillary expansion. The FE biomechanical analysis of the RME effect on the craniofacial complex in patients with unilateral cleft lip and palate was carried out in [21]. It is assumed that the orthodontic device is a rigid body [21]. To simulate the clinical situation the symmetrical displacements of the anchor teeth corresponding to a certain number of the orthodontic devices the screw turns were employed in [21]. In [21], it was noted that the similar simplifying assumptions were assumed in other analogous studies $[10,11,17]$. In [21] the FE evaluations of stresses were carried out without regard to the periodontal ligament, since due to the action of orthodontic appliance the anchor teeth completely overlay the periodontal gap. One of the conclusions of [21] was that the magnitudes of displacements on the normal side of skull are different from those on the skull side with the palate cleft. In accordance with [21] this may be caused by an asymmetrical disposition of the skull bone structures. One of the few FE studies on the comparative analysis of the stress distributions in the skull without cleft, in the skull with unilateral cleft and with 
bilateral cleft is [5]. In [5] action of the orthodontic appliance Quad-Helix on the maxilla was simulated without periodontal ligament. According to [5], the smallest expansion is observed between the control points for the skull without palate cleft, and the difference between the stress distributions in the skull without cleft and in the skull with cleft (unilateral or bilateral) during RME is quite substantial.

The aim of this study is a comparative analysis of the stresses patterns and displacements of bone structures for the skull without palate cleft and for the skulls with unilateral and bilateral palate cleft after activation of the Hyrax orthodontic device during RME. The feature of the present study is that the orthodontic device is a deformable rod construction with different dispositions of the screw relative to the palate. Therefore, another objective is to evaluate the effect of the screw location on the stresses and displacements distributions in the craniofacial complex with and without palate cleft. FE method was used to reach these goals.

\section{FE MODELING OF MAXILLARY EXPANSION}

\subsection{Solid models of skull, Hyrax device and anchor teeth}

Stereolithography (STL) model of the skull is developed using MIMICS 14.12 (Materialise BV, Leuven, Belgium) on the basis of 210 tomographic images of the dry cadaveric intact skull of an adult with a well-preserved alveolar bone and teeth. Models of the first and the second upper premolars (14, 15 and 24, 25 teeth), and the permanent molars (16 and 26 teeth) are also generated based on the tomographic data. Model of Hyrax orthodontic device is developed via SolidWorks 2010 (SolidWorks Corporation, USA). Four rods of orthodontic device are affixed to the plates with screw and to the crowns; another two rods are affixed to the crowns and are impacted on the second premolars (15 and 25 teeth). Simulation of the periodontal ligament is not carried out since the periodontal ligament has almost no effect on the stresses distribution in the craniofacial bones [22]. Cranium sutures are not accounted in the FE model. This is because in the adult human skull the sutures are partially or fully ossified [1].

\subsection{Boundary conditions. Geometrical and material parameters}

The FE skull model is fixed in the nodes located around of the foramen magnum [10, 13, 17]. Displacement of each plate of the orthodontic device is directed only transversely (along $x$-axis). Boundary conditions are indicated in Fig. 1.

Two constructions of the orthodontic device are considered: construction with disposition of rods and screws in a single horizontal (occlusal) plane (Model 1); construction with plates and screw shifted closer to the palate relative to the horizontal plane on $8 \mathrm{~mm}$ (Model 2). The geometrical dimensions of the orthodontic devices are identical, with the exception of lengths of the rods fastening the plates with first premolars and molars. Models 1 and 2 are fixed on the anchor teeth of the skull without cleft (SWC), on the skull with a unilateral cleft (SULC) and on the skull with bilateral cleft (SBLC). Elastic properties of materials for orthodontic device, skull bones and teeth are given in Table 1. 
Table 1 Mechanical properties of the materials

\begin{tabular}{lcc}
\hline Material & Elastic modulus, GPa & Poisson's ratio \\
\hline Steel (orthodontic device) & 200.0 & 0.3 \\
Cortical bone [20] & 13.7 & 0.3 \\
Trabecular bone [20] & 8.0 & 0.3 \\
Teeth [20] & 20.7 & 0.3 \\
\hline
\end{tabular}
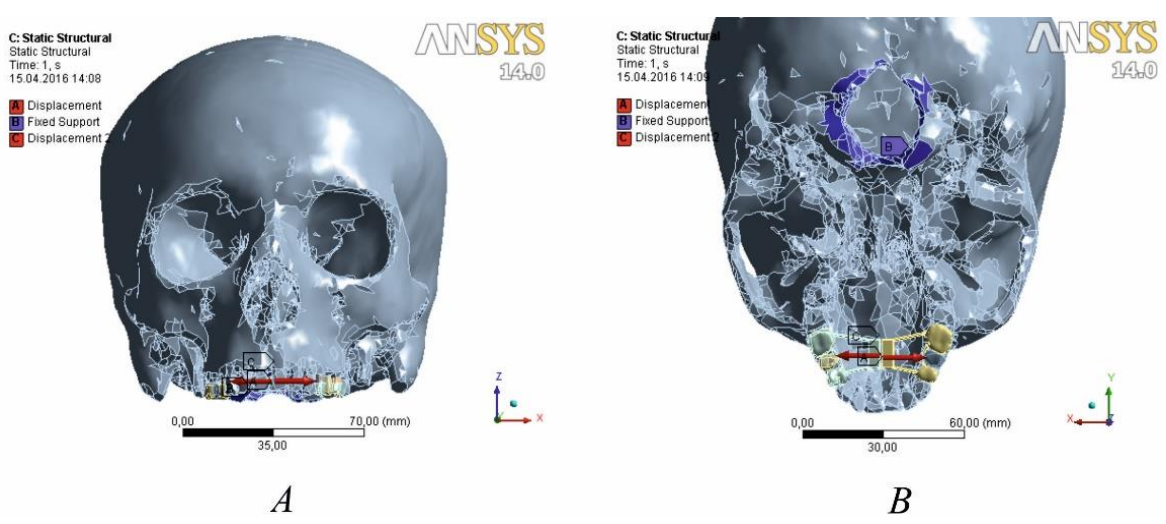

Fig. 1 Boundary conditions for the FE skull model ( $A$ is front view, $B$ is view from below): the boundary conditions, marked by $\mathrm{A}$ and $\mathrm{C}$ correspond to displacements of orthodontic device plates in transversal direction (along the $x$-axis); boundary condition, marked by $\mathrm{B}$ corresponds to skull fixing in nodes around foramen magnum

The lengths of the rods for Models 1 and 2 between the device plates and the crowns vary from $8.15 \mathrm{~mm}$ to $11.05 \mathrm{~mm}$ and $12.20 \mathrm{~mm}$ and $16.45 \mathrm{~mm}$, respectively. The length and width of the plates for Models 1 and 2 are $10.0 \mathrm{~mm}$ and $4.0 \mathrm{~mm}$, respectively; the cross-sectional radius of the rods is equal to $1.0 \mathrm{~mm}$, thickness of crowns is $0.2 \mathrm{~mm}$.

\subsection{Parameters of FE models}

The FE models of the skull are developed after processing the STL-model in 3-matic 6.1 MIMICS. Discrete skull model is converted via FE Modeler of ANSYS Workbench14 (ANSYS Inc., USA). FE meshing is made in automatic mode (Solid72 type elements are used). The number of elements and nodes for the FE models of skulls, anchor teeth and orthodontic devices are given in Table 2.

Table 2 Parameters of the FE models

\begin{tabular}{lcc}
\hline Model & Node number & Element number \\
\hline SWC & 77036 & 185302 \\
SULC & 24556 & 85087 \\
SBLC & 24494 & 85138 \\
Model 1 & 15918 & 7798 \\
Model 2 & 16410 & 8022 \\
\hline
\end{tabular}


Contacts between the crowns and the teeth, as well as between the maxilla and the teeth are 'Bonded' type without sliding and mutual penetration.

\section{FE ANALYSIS OF CRANIOFACIAL STRESSES}

The FE analysis of stresses (von Mises) and displacements for SWC, SULC and SBLC is carried out after activations of Models 1 and 2 by means of the transversal displacements of the orthodontic device plates. Transversal displacement of each plate of Models 1 and 2 is $0.2 \mathrm{~mm}$ (corresponding to the activation of the orthodontic device screw on a quarter turn $[2,8,15])$.

\subsection{Skull without cleft}

The stress patterns in SWC after activations of Models 1 and 2 are depicted in Figs. 2 and 3, respectively. In Figs. 2-7 the magnitudes of stresses are given in MPa.

Fig. 2 shows that the sufficient stresses in SWC after activation of Model 1 appear mostly in the maxilla. High stresses occur in the middle and bottom of the nasal cavity and the bottom of the left orbit. The stresses near only one orbit can be explained by the asymmetry of the craniofacial complex and asymmetric disposition of points of rods fixing on the crowns. The higher stresses in the left infraorbital foramen in comparison with the right one (see Fig. 2, A) are also obviously caused by this asymmetry.

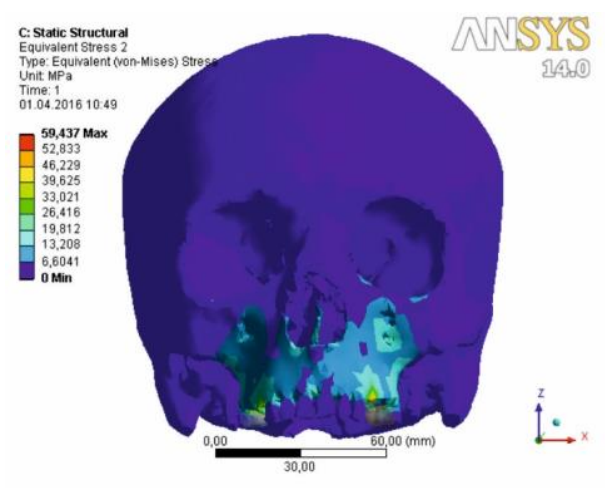

$A$

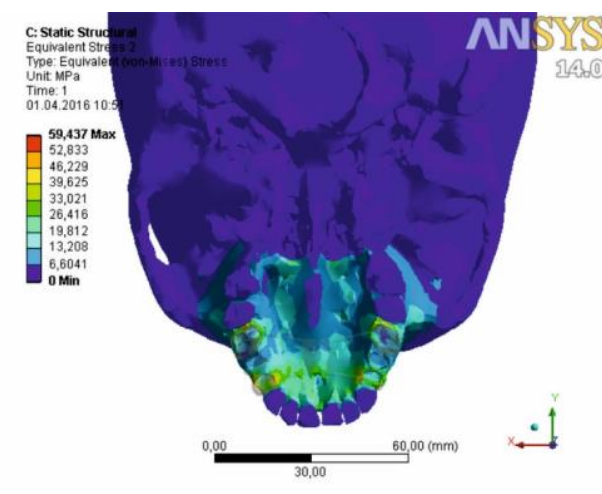

$B$

Fig. 2 Stress patterns in SWC after activation of Model 1: $A$ is pattern in front of skull; $B$ is pattern in base of skull

Stress distribution in SWC after activation of Model 2 significantly changes when compared to Model 1. Fig. 3 shows that the maxilla is loaded partially and the highest stresses are reduced approximately to $16.10 \mathrm{MPa}$. The region with nonzero stresses in the anterior part of SWC after activation of Model 2 displaces from the maxilla to the nasal cavity and zygomatic process. In the median palatine suture region there are almost no stresses; they only appear in the incisive bone (see Fig. 3, B). At the same time, the stresses are observed in the occipital bone near the foramen magnum and in the pterygoid plate, but almost no stresses exist in the median palatine suture. 


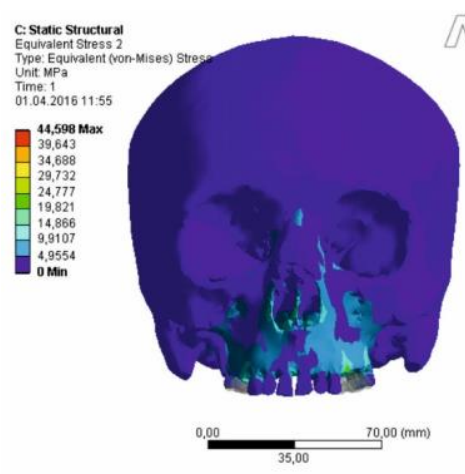

$A$

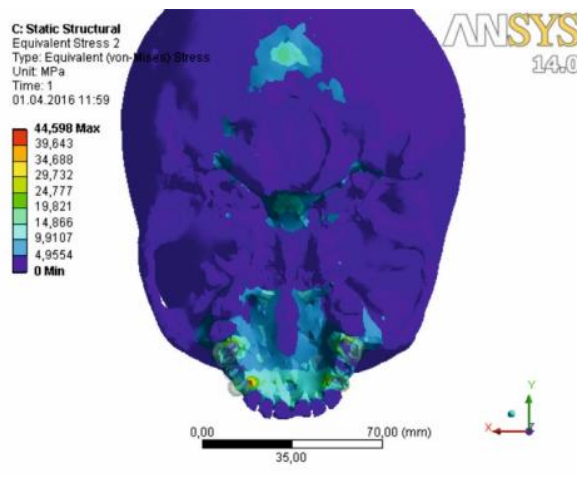

$B$

Fig. 3 Stress patterns in SWC after activation of Model 2:

$A$ is pattern in the front of the skull; $B$ is pattern in the base of the skull

The highest stresses in SWC after activation both of Models 1 and 2 occur in the alveolar bone surrounding the anchor teeth (see Figs. 2, $B$ and 3, B). Nonzero stresses also appear in the zygomatic arches after activation of Models 1 or 2 . If the screw of the orthodontic device is displaced to the palate, the region with nonzero stresses of the zygomatic arches increases.

\subsection{Skull with unilateral cleft}

The palate cleft is complete and passes on the level of second maxilla incisor. Figs. 4 and 5 depict the stresses patterns in SULC after activations of Models 1 and 2, respectively.
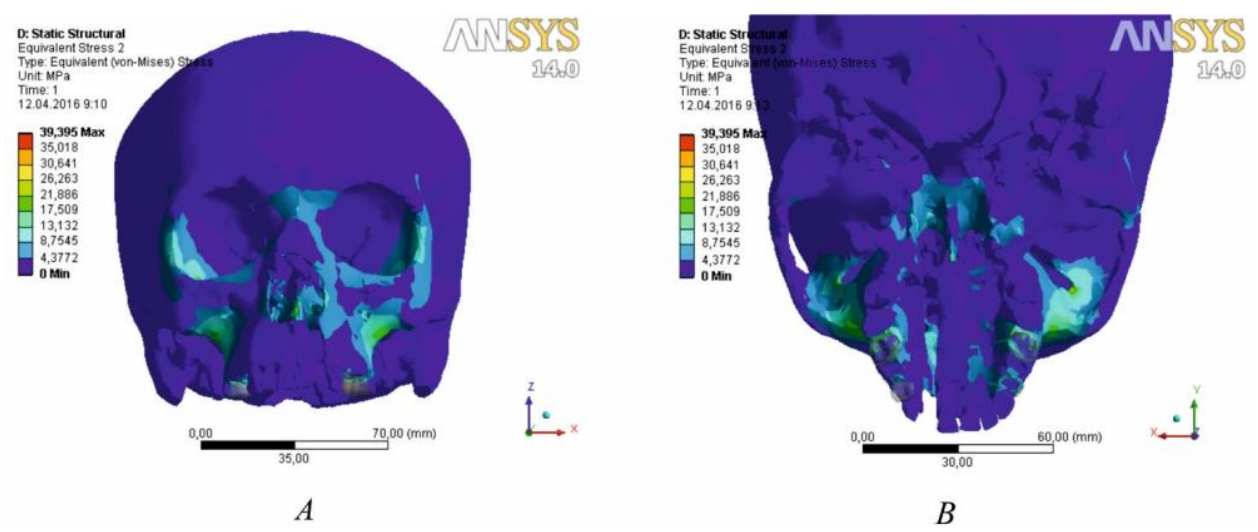

Fig. 4 Stress patterns in SULC after activation of Model 1: $A$ is pattern in front of skull; $B$ is pattern in base of skull

Fig. 4 shows that after activation of Model 1 high stresses occur in the maxilla bone, particularly in the zygomatic processes of the maxilla below the infraorbital foramen. 
High stresses appear in the regions of the nasal cavity and orbits, in the bone of frontal process of the maxilla on the cleft side, in the nasal bones as well as in the regions of the fronto-nasal, inter-nasal and inter-nasal-maxillary sutures. The high stresses in the base of the skull are distributed through the lateral and medial pterygoid plate to the pharyngeal tubercle. Stresses also occur in the region surrounding the silcus of the auditory tube. This indicates that the RME can have a significant impact on the increase of the nasal cavity dimension and improve nasal breathing [1, 11, 12, 16, 23] just as it leads to changes of the auditory conductivity in patients with cleft palate. The short-term and long-term impacts of maxillary expansion on the auditory conduction are described in [14].
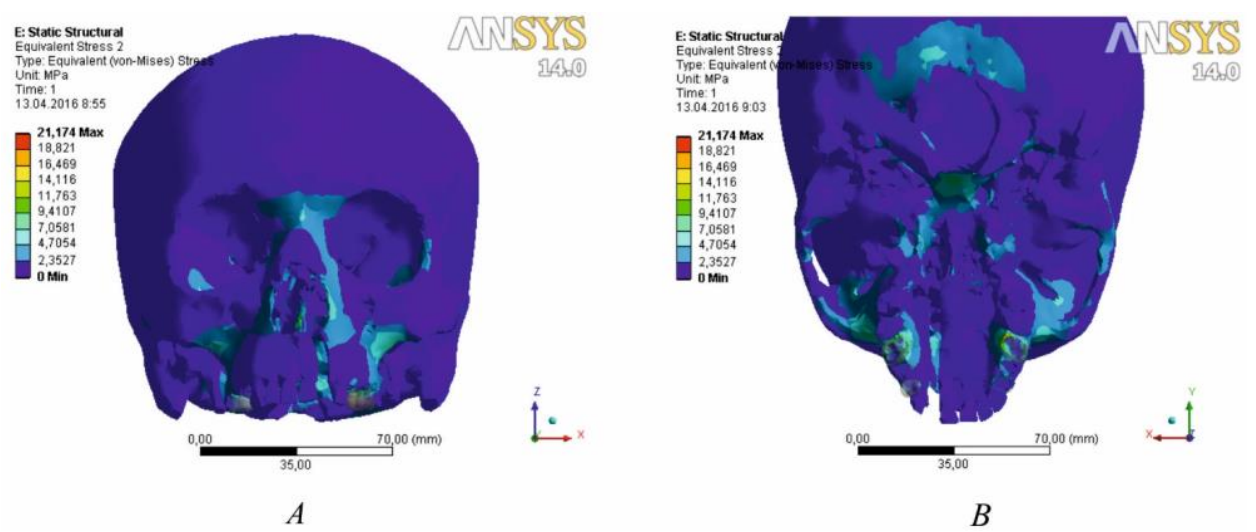

Fig. 5 Stress patterns in SULC after activation of Model 2: $A$ is pattern in front of skull; $B$ is pattern in base of skull

Figs. 4 and 5 indicate that the stresses patterns in the anterior part of SULC slightly differ after activations of Models 1 and 2. However, the maximum stresses in SULC after the activation of Model 2 are significantly lower than the maximum stresses in SULC after activation of Model 1. At the same time, high stresses occur in the zygomatic process after activation of both Models 1 and 2. Significant stresses appear at the skull base in the foramen magnum region after activation of Model 2 (see Fig. 5, B). In the palatal region of the maxilla after activations of Models 1 and 2 there are almost no stresses, with the exception of small regions of the alveolar processes.

\subsection{Skull with bilateral cleft}

The bilateral palate cleft is complete and passes at the level of the second maxilla incisors on the left and the right sides of the skull. Figs. 6 and 7 depict the stresses patterns in SBLC after activations of Models 1 and 2.

It is seen from Fig. 6, $A$ and Fig. 7, $B$, the stresses patterns in the anterior part of SBLC after activations of Models 1 and 2 are almost the same. At the same time, the magnitudes of stresses in SBLC vary significantly in dependence on Model 1 or Model 2. After activation of Model 1 the maximum stresses are approximately equal to $24.0 \mathrm{MPa}$, while after activation of Model 2 the maximum stresses are approximately equal to $1.55 \mathrm{MPa}$. The highest stresses in SBLC after activations of Models 1 and 2 are observed in the zygomatic 
and alveolar processes of the maxilla and in the zygomatic bone. Also, the high stresses in SBLC compared with SWC and SULC after activation of Model 1 occur in the sphenoid and nasal bones, as well as in the frontal processes of the maxilla.

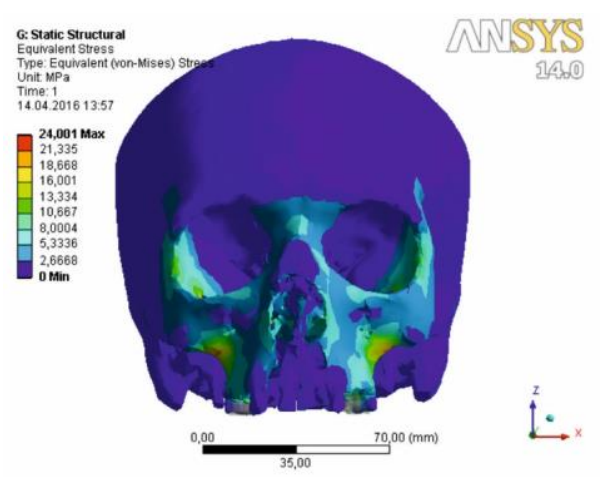

$A$

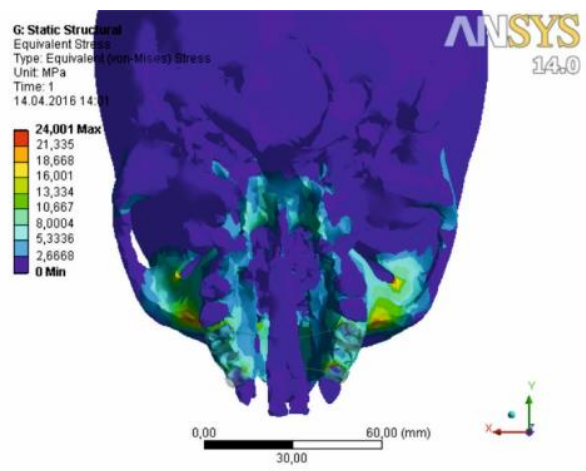

$B$

Fig. 6 Stress patterns in SBLC after activation of Model 1: $A$ is pattern in front of skull; $B$ is pattern in base of skull

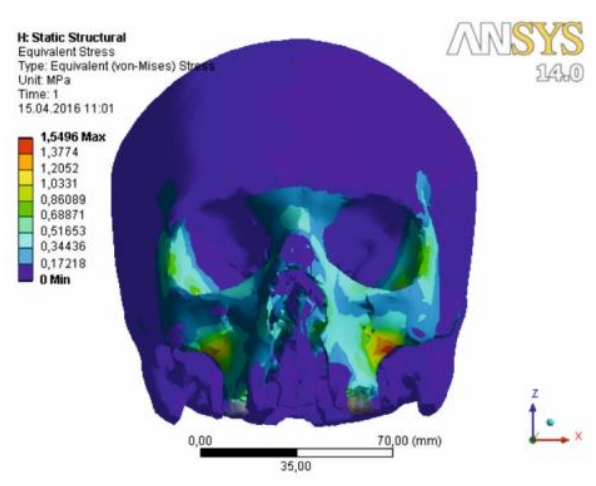

A

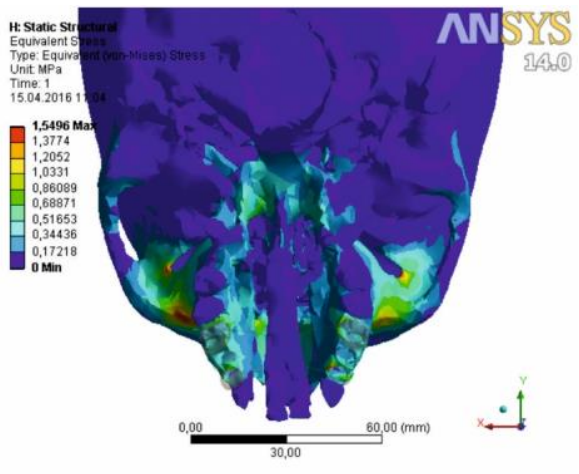

$B$

Fig. 7 Stress patterns in SBLC after activation of Model 2:

$A$ is pattern in front of skull; $B$ is pattern in base of skull

The stress patterns are almost the same in the base of SBLC after activation of Models 1 or 2 (see Fig. 6, $B$ and Fig. 7, $B$ ). However, the region with the nonzero stresses of the pharyngeal tubercle is larger after activation of Model 2 compared with Model 1. The magnitudes of the stresses in SBLC after activation of Model 2 (maximal stresses approximately are $1.21 \mathrm{MPa}$, see Fig. $7, B$ ) are significantly less than the stresses in SBLC after activation of Model 1 (maximal stresses approximately equal to $16.0 \mathrm{MPa}$, see Fig. 6, $B$ ). 


\section{FE ANALYSIS OF DISPLACEMENTS}

\subsection{Intact skull}

The vector fields of the total displacements and the distributions of displacements along the coordinate axes for SWC points after activations of Models 1 and 2 are depicted in Figs. 8 and 9. Displacements along the $x$-, $y$ - and $z$-axes are the transversal, sagittal and vertical displacements, respectively. The magnitudes of displacements in Figs. 8-13 are given in $\mathrm{mm}$.

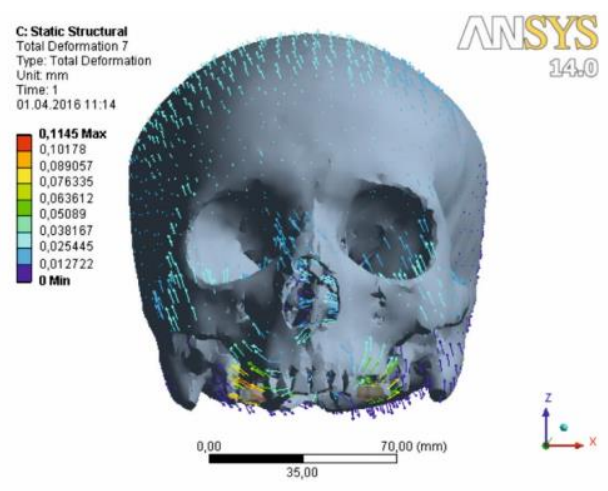

A

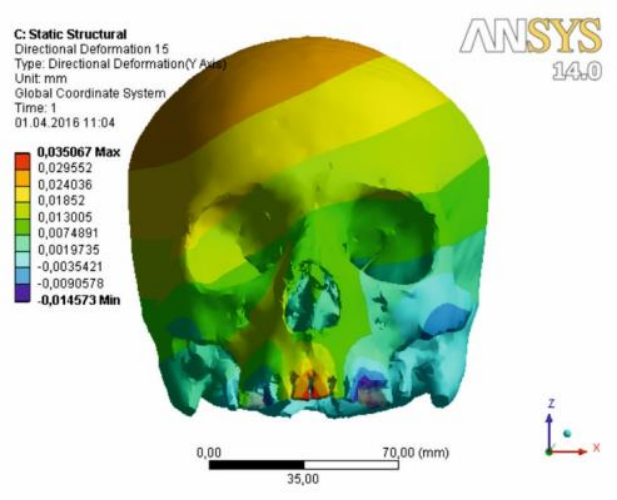

C

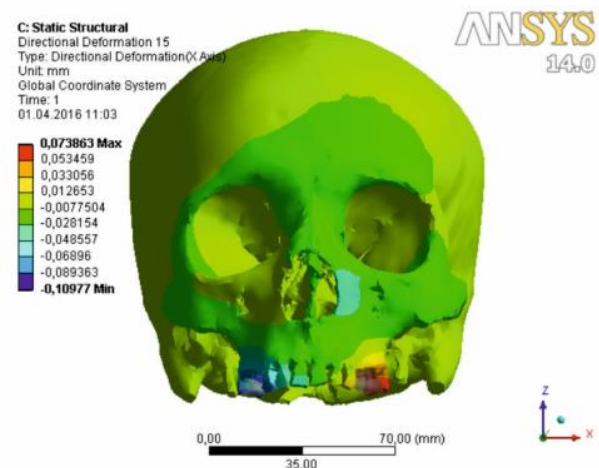

B

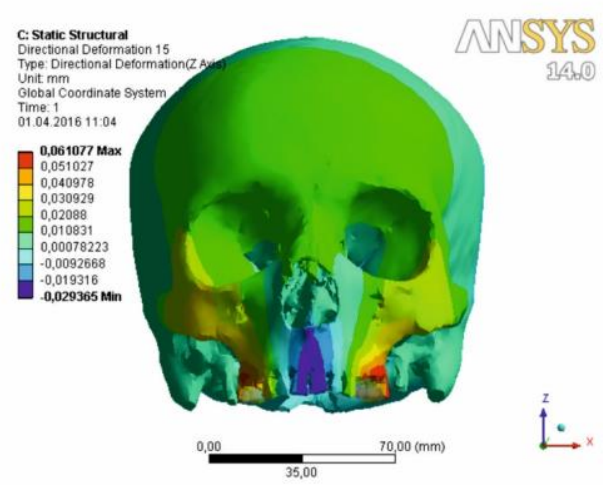

D

Fig. 8 Displacements of SWC points after activation of Model 1: $A$ is vector field of total displacements; $B$ is distribution of transversal displacements; $C$ is distribution of sagittal displacements; $D$ is distribution of vertical displacements

It is seen from Fig. 8 that the two maxilla halves after activation of Model 1 turn relative to the horizontal axis passing approximately through the nasal aperture region and parallel to the $y$-axis. The highest components of the total displacements are transversal and vertical displacements (see Fig. 8, $B$ and $D$ ). The vertical displacements of SWC 
points in the region of the anterior incisors and nasal aperture are directed downwards, while the displacements of rest of the skull are directed upwards.

The sagittal displacements of SWC points after activation of Model 1 are the smallest of the three components of total displacements (see Fig. 8, C). The upper part of SWC and anterior region of the maxilla after the activation of Model 1 are moved backwards in the horizontal direction, while the rest of the maxilla and zygomatic arches slightly moves forwards.

It is seen from Fig. 9 that the total displacement of SWC points after activation of Model 2 are directed, basically, along $z$-axis so that the anterior part of SWC is moved downwards, while the posterior part of SWC is moved upwards, which leads to rotation of SWC counterclockwise (relative to the positive direction $z$-axis). This conclusion is confirmed by the distribution of vertical displacements (see Fig. 9, D). Fig. 9, $A$ and Fig. 9, $D$ indicate that the maximal (in absolute value) vertical displacements of SWC points after activation of Model 2 are directed downwards in the anterior part. Such direction of the vertical displacements corresponds to the distribution of the sagittal displacements (see Fig. 9, C). The displacements distributions in Fig. 9 show that SWC after activation of Model 2 is rotated relative a horizontal axis without passing through the cranium itself. Axis of SWC rotation is located in the region of the foramen magnum and pharyngeal tubercle and parallel to the $x$-axis.

\subsection{Skull with unilateral cleft}

The vector field of the total displacements and distributions of the transversal, sagittal and vertical displacements of SULC points after activation of Models 1 and 2 are shown in Figs. 10 and 11.

Fig. 10, $A$ and Fig. 10, $B$ show that the transversal displacements are the largest components of SULC total displacements after activation of Model 1. The vector field of the total displacements and the transversal displacements distribution are almost symmetrical (see Fig. 10, $A$ and Fig. 10, B). For instance, the highest magnitudes of oppositely directed transversal displacements are approximately equal to $0.204 \mathrm{~mm}$ and $2.0 \mathrm{~mm}$ respectively. Fig. 10, $C$ and Fig. 10, $D$ show that the distributions of the sagittal and vertical displacements of SULC points after activation of Model 1 are almost symmetrical as well. The maximum values of these displacements differ slightly. Distribution of sagittal displacement shows that the frontal bone deflects backwards, while the maxillary region containing the inter-maxillary suture and the cleft is moved forwards (see Fig. 9, C). The vertical displacements of SULC anterior part are directed upwards, while the displacements the points on SULC posterior part are directed downwards (see Fig. 10, D). The largest vertical displacements are observed for points of the zygomatic processes and the zygomatic bones.

The maximal total displacement of SULC points after activation of Model 2 and Model 1 (see Fig. 10, $A$ and Fig. 11, A) are almost the same. However, basically, the total displacements of SULC points after activation of Model 2 are directed downwards unlike the directions of the total displacements of SULC points after activation of Model 1. 


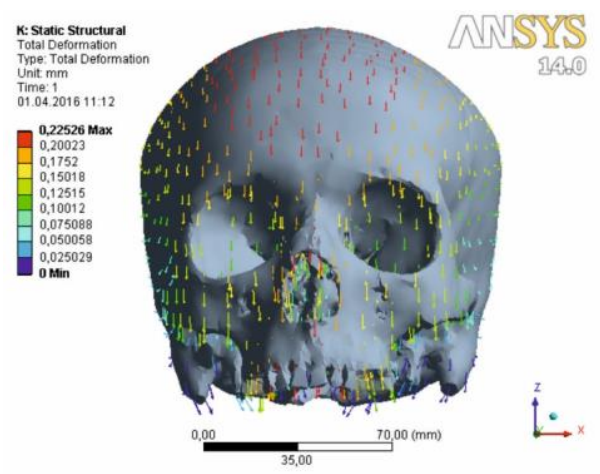

A

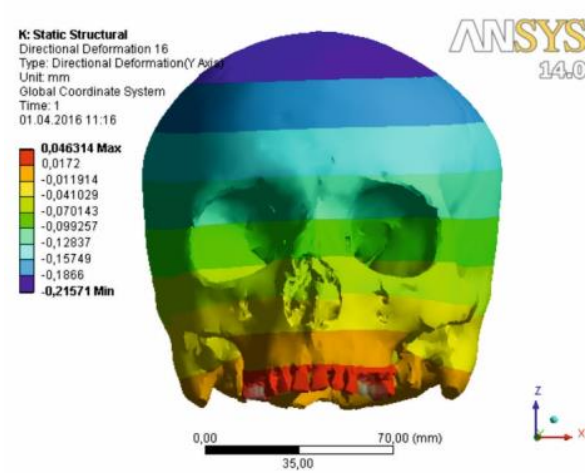

C

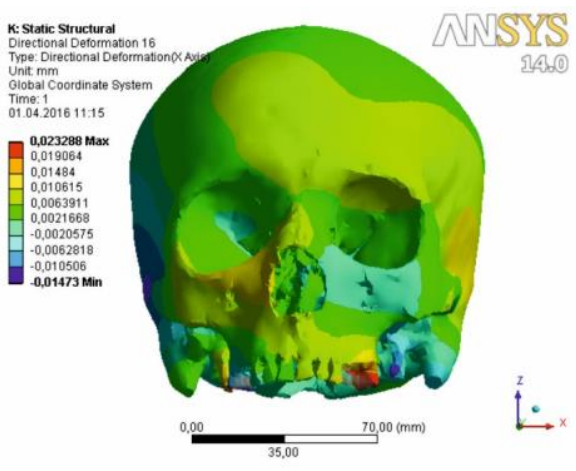

$B$

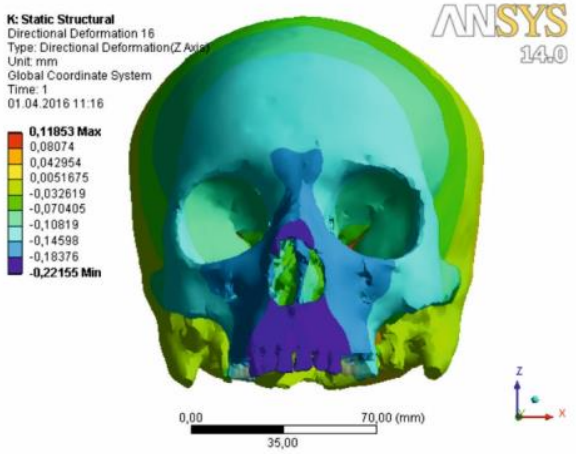

$D$

Fig. 9 Displacements of SULC points after activation of Model 2: $A$ is vector field of total displacements; $B$ is distribution of transversal displacements; $C$ is distribution of sagittal displacements; $D$ is distribution of vertical displacements

After activation of Model 2 the maximal transversal displacements (in dependence on direction) are equal to $0.0684 \mathrm{~mm}$ and $0.0536 \mathrm{~mm}$ (see Fig. 11, $B$ ), which is significantly less than the maximal transversal displacements of SULC points after activation of Model 1. Distributions of sagittal displacement of SULC points after activation of Models 1 (Fig. $10, C$ ) and 2 (Fig. 11,C) significantly differ from each other. According to Fig. 11, $C$, the SULC anterior part is moved backwards while the bone structures of maxilla and zygomatic bone are moved forwards. The sagittal displacements direction of SULC points indicates its anti-clockwise rotation (from the positive direction of the $x$-axis). This is confirmed by the distribution of the vertical displacements (see Fig. 11, D). According to Fig. 11, D, the facial and occipital parts and of SULC are moved downwards and upwards, respectively. Considering symmetrical distribution of the sagittal and vertical displacements, it can be concluded that after activation of Model 2 SULC is rotated in $y z-$ plane relative horizontal axis located approximately above the foramen magnum and parallel to the $x$-axis. 

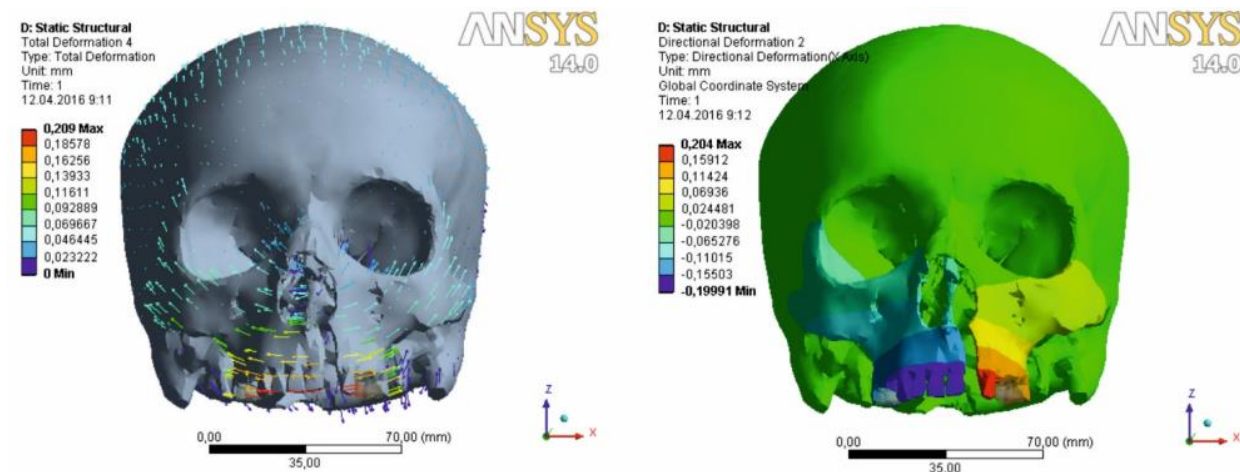

A

B
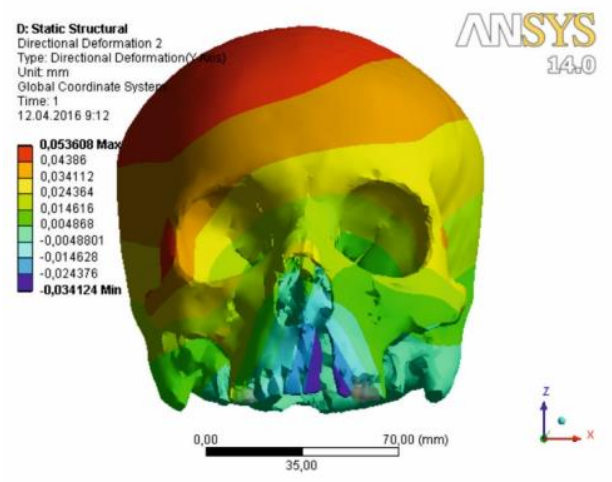

C

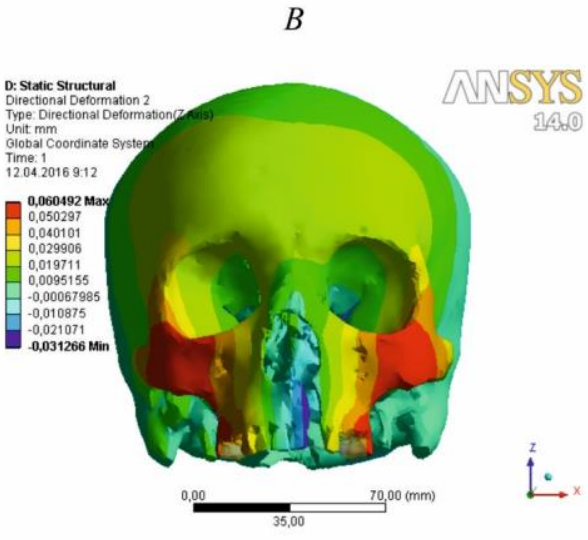

$D$

Fig. 10 Displacements of SULC points after activation of Model 1: $A$ is vector field of total displacements; $B$ is distribution of transversal displacements; $C$ is distribution of sagittal displacements; $D$ is distribution of vertical displacements

Note that the after activations of Models 1 and 2 the transversal displacements of SULC points on the side with cleft are larger than the displacements of the normal side. Distributions of the transversal displacements of SULC (see Fig. 10, $B$ and Fig. 11, $B$ ) also indicate asymmetry of the cranium displacements. This is consistent with the results of the [16] that the displacements of side of the skull with cleft are larger than those of the normal side.

\subsection{Skull with bilateral cleft}

The vector field of the total displacements and distributions of the transversal, sagittal and vertical displacements in SBLC after activations Models 1 and 2 are shown in Figs. 12 and 13. 

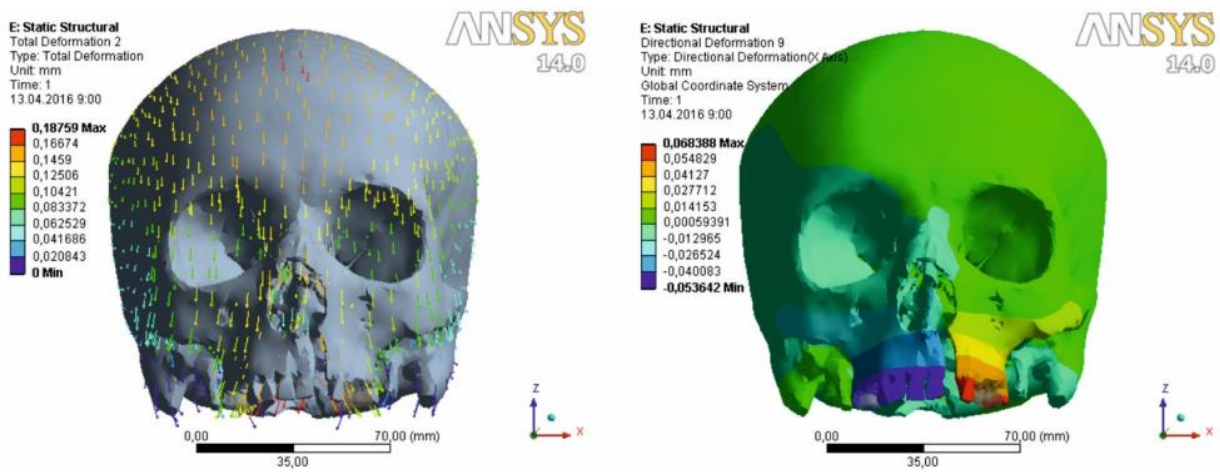

$A$

$B$

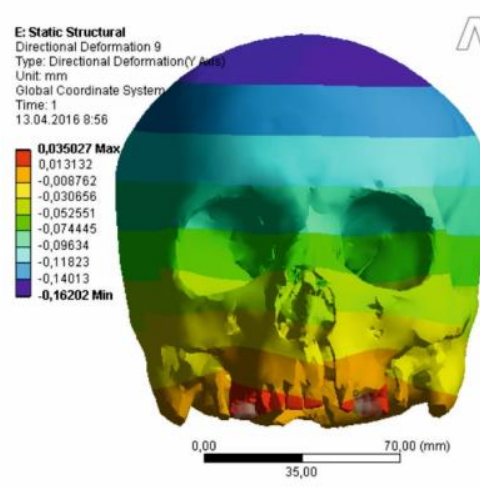

MNSIS

$C$

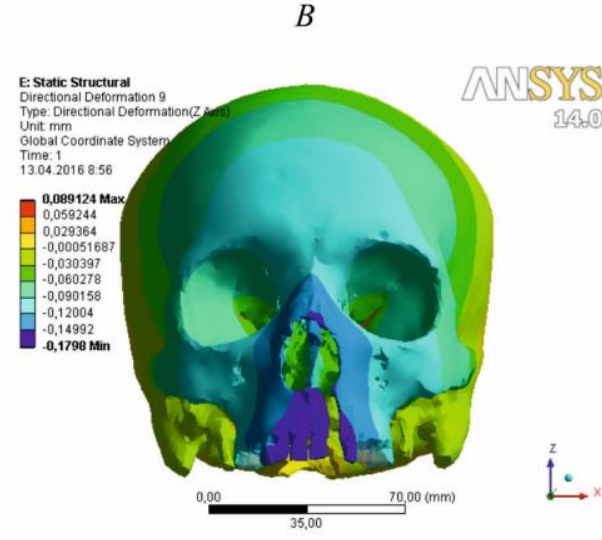

$D$

Fig. 11 Displacements of SULC points after activation of Model 2: $A$ is vector field of total displacements; $B$ is distribution of transversal displacements; $C$ is distribution of sagittal displacements; $D$ is distribution of vertical displacements

It is seen from Figs. 12, $A$ and 13, $A$, that the directions of total displacements of SBLC points are almost identical after activations of both Models 1 and 2, while the magnitudes of the total displacements for these two cases are significantly different. The maximal component of the total displacement after activations of Models 1 and 2 is transversal. The region of SBLC with the inter-maxillary suture does not move in the horizontal direction after activation of Model 1. The transversal displacements of this region after activation of Model 2 are very small (see Fig. 13, B). Sagittal and vertical displacements of SBLC points are significantly less than transversal displacements after activation of Models 1 and 2.

Directions of the sagittal and vertical displacements indicate that there is a slight rotation of SBLC in $y z$-plane clockwise (relative to the positive direction of the $x$-axis); the angle of SBLC rotation is larger after activation of Model 1 compared with Model 2. The SBLC rotation axis after activation of Model 2 is located in the foramen magnum region, and it is slightly displaced towards the occipital bone. 


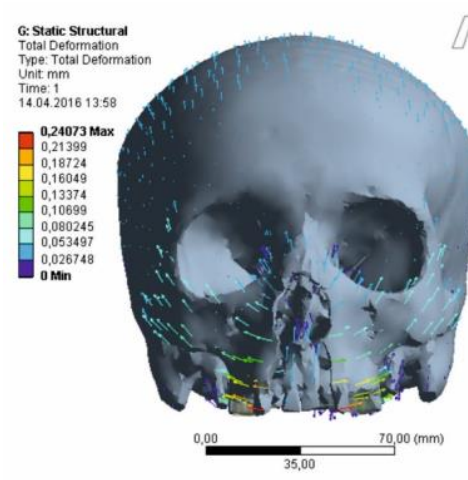

A

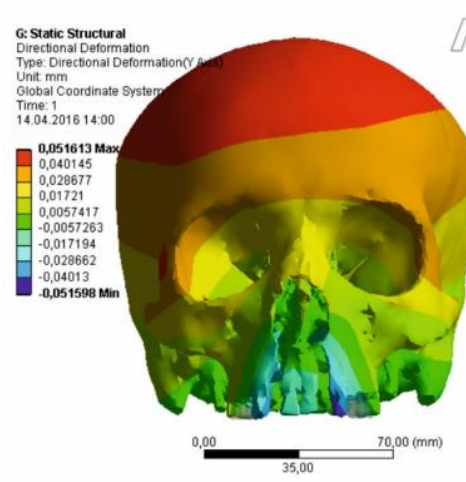

$C$
NNSTIS
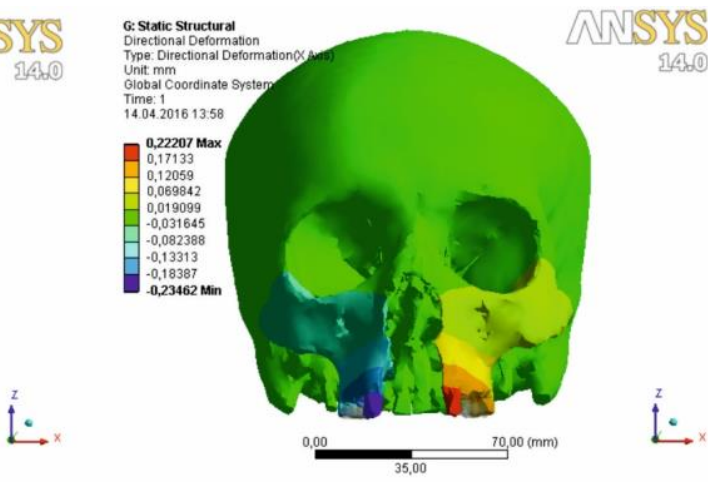

$B$

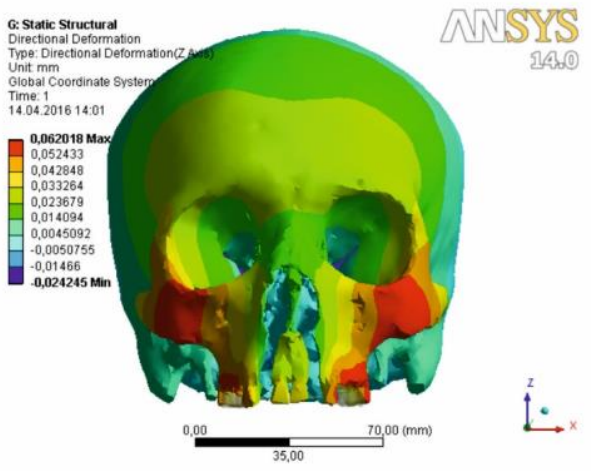

$D$

Fig. 12 Displacements of SBLC points after activation of Model 1: $A$ is vector field of total displacements; $B$ is distribution of transversal displacements; $C$ is distribution of sagittal displacements; $D$ is distribution of vertical displacements

\section{DISCUSSION}

Higher stresses in SWC, SULC SBLC occur after activation of Model 1 compared with Model 2. The smallest difference (in 1.86 times) between stresses after activations of Models 1 and 2 is observed in SULC. The highest difference (in 15.5 times) between the stresses is in SBLC after activations of Models 1 and 2. It should be noted that very low stresses appear in SBLC after activation of Model 2 (maximal magnitude of stresses is equal to $1.55 \mathrm{MPa}$ ).

The obtained results indicate that the regions of maximal stresses in SWC, SULC and SBLC, regardless of the orthodontic device model, occur in the alveolar processes of the maxilla and in the zygomatic bone (except SWC after activation of Model 2). Displacement of the orthodontic device screw to the palate leads to redistribution of stresses in SWC from the maxilla to the nasal cavity and to the foramen magnum. The most complicated distribution of stresses is observed in SULC after the activation of Models 1 and 2. In these 
cases, sufficiently high stresses propagate to many bone structures. Following displacement of the orthodontic device screw to the palate, the stresses in SWC and SULC are transferred to the foramen magnum and the pharyngeal tubercle. The orthodontic device construction has almost no effect on the stresses distribution in SBLC bone structures. Stresses in the sphenoid bone occur in SULC as well as in SBLC after activation of Model 2. This indicates the expediency of the osteotomy for reducing of resistance of the sphenoid bone (and the sphenoid plate) during RME.

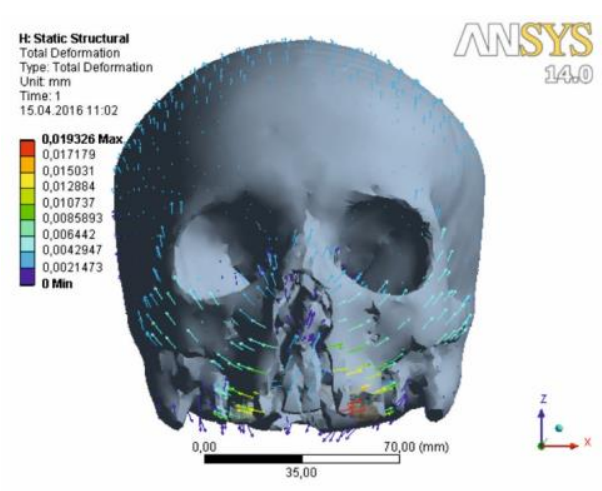

A

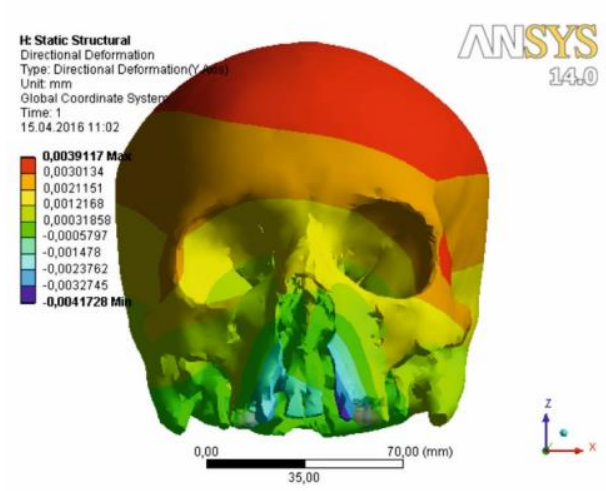

C

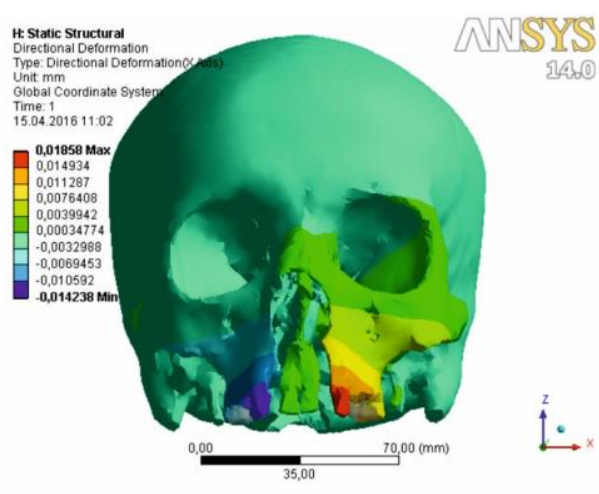

$B$

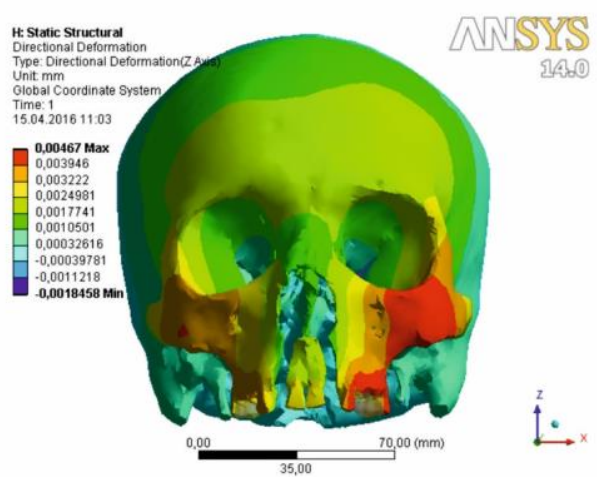

$D$

Fig. 13 Displacements of SBLC points after activation of Model 2: $A$ is vector field of total displacements; $B$ is distribution of transversal displacements; $C$ is distribution of sagittal displacements; $D$ is distribution of vertical displacements

Displacements of SBLC and SWC points after activation of Model 1 are some of the smallest. The highest total displacements are observed in SBLC after activation of Model 1. Also, the high displacements of SWC points are observed after activation of Model 2. The maximal displacements of SULC points are approximately the same after activations of both Models 1 and 2. At the same time, the transversal displacements of SWC points after activation of Model 1 can reach more than $91 \%$ of its total displacement, and $95 \%$ of the total displacements of SULC and the SBLC. The highest transversal, sagittal and 
vertical displacements are $11 \%, 92 \%$ and $97 \%$ of the maximal total displacements, respectively. This indicates that SWC moves forwards and downwards after the activation of Model 2. Similar ratios $(13 \%, 74 \%$, and $92 \%)$ between the maximal transversal, sagittal, vertical displacements and maximal total displacements of SULC points are observed after activation of Model 2. SBLC points displace significantly in the transversal direction after activations of Models 1 and 2. Magnitudes of the transversal displacement of some SBLC points coincide with the total displacement of the same points; the highest sagittal and vertical displacements are not more than $5 \%$ of the maximum total displacements.

Acknowledgements: This paper is the result of the implementation of the project: "Trans-Atlantic Micromechanics Evolving Research: Materials containing inhomogeneities of diverse physical properties, shapes and orientations» supported by FP7-PEOPLE-2013-IRSES Marie Curie Action «International Research Staff Exchange Scheme».

\section{REFERENCES}

1. Boryor, A., Geiger, M., Hohmann, A., Wunderlich, A., Sander, Ch., Sander, F.M., Sander, F.G., 2008, Stress distribution and displacement analysis during an intermaxillary disjunction -- A three-dimensional FEM study of a human skull, Journal of Biomechanics, 41 pp. 376-382.

2. Chaconas, S.J., Caputo, A.A., 1982, Observation of orthopedic force distribution produced by maxillary orthodontic appliances, American Joural of Orthodontics, 82 pp. 492-501.

3. Gautam, P., Valiathan, A., Adhikari, R., 2007, Stress and displacement patterns in the craniofacial skeleton with rapid maxillary expansion: a finite element method study, American Journal of Orthodontics and Dentofacial Orthopedics, 132 pp.5.e1-5.e11.

4. Gautam, P., Zhao, L., Patel, P., 2011, Biomechanical response of the maxillofacial skeleton to transpalatal orthopedic force in a unilateral palatal cleft, Angle Orthodontist, 81 (3) pp. 503-509.

5. Holberg, C., Holberg, N., Schwenzer, K., Wichelhaus, A., Rudzki-Janson, I., 2007, Biomechanical analysis of maxillary expansion in CLP patients, Angle Orthodontist, 77 pp. 280-287.

6. Holberg, C., Steinhäuser, S., Rudzki-Janson, I., 2007, Rapid maxillary expansion in adults: cranial stress reduction depending on the extent of surgery, European Journal of Orthodontics, 29 pp. 31-36.

7. Holberg C., Steinhäuser S., Rudzki, I., 2007, Surgically assisted rapid maxillary expansion: midfacial and cranial stress distribution, American Journal of Orthodontics and Dentofacial Orthopedics, 132 pp. 776-782.

8. Isaacson, R.J., Murphy, T.D., 1964, Some effects of rapid maxillary expansion in cleft lip and palate patients, Angle Orthodontist, 34 pp. 143-154.

9. Isaacson, R.J., Wood, J.L., Ingram, A.H., 1964, Forces produced by rapid maxillary expansion, Part I and II // Angle Orthodonics, 34 pp. 256-270.

10. Iseri, H., Tekkaya, A.E., Öztan, Ö., Bilgiç, S., 1998, Biomechanical effects of rapid maxillary expansion on the craniofacial skeleton, studied by the finite element method, European Journal of Orthodontics, 20 pp. 347-356

11. Jafari, A., Shetty, K.S., Kumar, M., 2003, Study of stress distribution and displacement of various craniofacial structures following application of transverse orthopedic forces - a three dimensional FEM study, Angle Orthodontist, 73 pp. 12-20.

12. Lee, H., Ting, K., Nelson, M., Sun, N., Sung, S.-J., 2009, Maxillary expansion in customized finite element method models, American Journal of Orthodontics and Dentofacial Orthopedics, 136 pp. 367-374.

13. Ludwig, B., Baumgaertel, S., Zorkun, B., Bonitz, L., Glasl, B., Wilmes, B., Lisson, J., 2013, Application of a new viscoelastic finite element method model and analysis of miniscrew-supported hybrid hyrax treatment, American Journal Orthodontics and Dentofacial Orthopedics, 143 pp. 426-435.

14. McGuinness, N.J., McDonald, J.P., 2006, Changes in natural head position observed immediately and one year after rapid maxillary expansion, European Journal of Orthodontics, 28 pp. 126-134

15. Memikoglu, T.U.T., Iseri, H., 1999, Effects of a bonded rapid maxillary expansion appliance during orthodontic treatment, Angle Orthodontics, 69 (3) pp. 251-256. 
16. Pan., X., Qian Yu., Yu, Q., Wang, D., Tang, Y., Shen, G., 2007, Biomechanical effects of rapid palatal expansion on the craniofacial skeleton with cleft palate: a three-dimensional finite element analysis, Cleft Palate Craniofacial Journal, 44 pp. 149-154.

17. Provatidis, C., Georgiopoulos, B., Kotinas, A., McDonald, J.P., 2007, On the FEM modeling of craniofacial changes during rapid maxillary expansion, Medical Engineering and Physics, 29 pp. 566-579.

18. Romanyk, D.L., Lagravere, M.O., Toogood, R.W., Major, P.W., Carey, J.P., 2010, Review of maxillary expansion appliance activation methods: engineering and clinical perspectives, Journal of Dental Biomechanics, DOI:10.4061/2010/496906

19. Sander, C., Hüffmeier S., Sander, F.M., Sander, F.G., 2006, Initial results regarding force exertion during rapid maxillary expansion in children // Journal of Orofacial Orthopedics, 67 pp. 19-26.

20. Tanne, K, Hiraga, J, Kakiuchi, K, Yamagata, Y, Sakuda, M., 1989, Biomechanical effect of anteriorly directed extraoral forces on the craniofacial complex: a study using the finite elements method. American Journal of Orthodontics and Dentofacial Orthopedics, 95(3) pp. 200-207.

21. Wang, D., Cheng, L., Wang, Ch., Qian Yu., Pan X., 2009, Biomechanical analysis of rapid maxillary expansion in the UCLP patient, Medical Engineering and Physics, 31 pp. 409-417.

22. Wood, S.A., Strait, D.S., Dumont, E.R., Ross, C.F., Grosse, I.R., 2011, The effects of modeling simplifications on craniofacial finite element models: The alveoli (tooth sockets) and periodontal ligaments, Journal of Biomechanics, 44 pp. 1831-1838.

23. Zimring, J.F., Isaacson, R.J., 1965, Forces produced by rapid maxillary expansion III. Forces present during retention, Angle Orthodontist, 35 pp. 178-186. 